\title{
Relativistic impulse dynamics
}

\author{
Stanley M. Swanson* \\ Department of Biochemistry and Biophysics, Texas A\&M University, College Station, Texas 77843-2128, USA
}

(Received 10 June 2010; revised manuscript received 31 May 2011; published 18 August 2011)

\begin{abstract}
Classical electrodynamics has some annoying rough edges. The self-energy of charges is infinite without a cutoff. The calculation of relativistic trajectories is difficult because of retardation and an average radiation reaction term. By reconceptuallizing electrodynamics in terms of exchanges of impulses rather than describing it by forces and potentials, we eliminate these problems. A fully relativistic theory using photonlike null impulses is developed. Numerical calculations for a two-body, one-impulse-in-transit model are discussed. A simple relationship between center-of-mass scattering angle and angular momentum was found. It reproduces the Rutherford cross section at low velocities and agrees with the leading term of relativistic distinguishable-particle quantum cross sections (Møller, Mott) when the distance of closest approach is larger than the Compton wavelength of the particle. Magnetism emerges as a consequence of viewing retarded and advanced interactions from the vantage point of an instantaneous radius vector. Radiation reaction becomes the local conservation of energy-momentum between the radiating particle and the emitted impulse. A net action is defined that could be used in developing quantum dynamics without potentials. A reinterpretation of Newton's laws extends them to relativistic motion.
\end{abstract}

DOI: 10.1103/PhysRevE.84.026611

PACS number(s): 03.50.De, 45.50.-j, 45.20.-d, 03.65.Nk

\section{INTRODUCTION}

Conventional dynamics describes interactions with forces and fields; impulses are an afterthought. In contrast, impulse dynamics does not use forces or fields or differential equations; it models interactions as a continual exchange of impulses. But why bother with impulses? The familiar forces, fields, and potentials are not always a satisfactory foundation for dynamics. There are fundamental problems in classical electrodynamics that impulse dynamics successfully addresses or avoids.

The divergent electrostatic self-energy of charges is avoided because there are no self-fields; impulse dynamics deals only with the interaction between particles. The calculation of two-particle trajectories does not involve hard to solve retarded differential equations; it is algebraic and straightforward, although numerically challenging. Previous numerical studies of relativistic trajectories with retarded forces [1] have invoked radiation reaction to get small energy losses rather than an unphysical energy gain when using Lorentz forces without radiation reaction. Impulse dynamics gives consistent trajectories without introducing true radiation at all. Radiation reaction can be dealt with exactly and immediately by subtracting the energy and momentum of a radiated null impulse at the interaction vertex.

Surprisingly, the straightforward approach of impulse dynamics solves or avoids some of the problems at the foundations of classical electrodynamics. However, without potentials, the familiar Hamiltonian-Lagrangian formulation of quantum mechanics is unavailable. An approach similar to Feynman's path integrals may be possible, since our treatment

\footnotetext{
*Present address: 3604 Cavitt Ave., Bryan, Texas, 77801-4407, stanswanson@tamu.edu.

Published by American Physical Society under the terms of the Creative Commons Attribution 3.0 License. Further distribution of this work must maintain attribution to the author(s) and the published article's title, journal citation, and DOI.
}

of impulse and particle energies and momenta is quantumlike. We are able to show how the classical action along a trajectory defines a Coulomb phase shift.

\section{DYNAMIC MODEL}

The basic dynamical system is two free particles with a photonlike null impulse in transit between them. An impulse is absorbed by one particle and another impulse immediately re-emitted toward the future position of the other particle where it will be absorbed. This future position is known because the absorbing particle is travelling with constant velocity between interaction vertices. The interaction is expressed by a continual exchange of impulses.

Except at the instant of absorption and emission, the total four-momentum of the system is

$$
p_{\text {total }}=p+q+k=p_{n-2, n}+p_{n-1, n+1}+k_{n-1, n} .
$$

To make momenta and events unambiguous, we introduce the convention of labeling momenta by the vertex numbers of the events at the start and end of that segment of the motion, as illustrated in Fig. 1. The last part of Eq. (1) makes the momentum choices explicit for the time interval $t_{n-1}<t<t_{n}$. For any given source (emitting) vertex $n$, we retain $x$ and $p$ to denote the position and four-momentum but change notation to $y$ and $q$ for the absorbing vertex variables. Because particles move with their free velocities and the impulses are proportional to the null vector between two vertices, the space-time location of the absorbing vertex $y=x_{n+1}$ is easily computed from the emitting vertex $x=x_{n}$, the absorbing particle momentum $q=p_{n-1, n+1}$, and the vertex $x_{n-1}$ from which $q$ originates. By requiring continuity of energy-momentum flow at an interaction vertex,

$$
\begin{aligned}
p_{\text {vertex }} & =p_{i}+k_{i}=p_{f}+k_{f} \\
& =p_{n-2, n}+k_{n-1, n}=p_{n, n+2}+k_{n, n+1},
\end{aligned}
$$

we are assured of energy-momentum conservation at all times. 


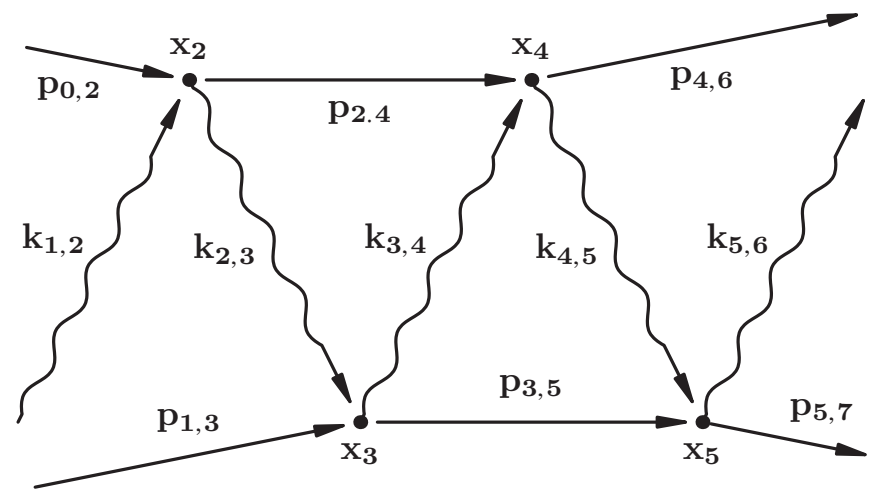

FIG. 1. Labeling scheme for vertices and momenta. These are physical space-time trajectories; this is not a Feynman ladder diagram. Particle $a$, with mass $m_{a}$, has even subscripts and particle $b$, with mass $m_{b}$, has odd subscripts. Impulses (k) are continually exchanged between successive vertices. In our calculations, the particles move in roughly opposite directions, but that would obscure the labeling of the impulses (photons).

Only minimum smoothness is imposed on trajectories. Motion is continuous, but velocities are either constant or change abruptly at interaction vertices. We give up differentiability. Coordinates and dynamical variables satisfy the invariance and covariance requirements of special relativity.

We now define a relativistic impulse that reduces to a Coulomb-like interaction in the zero velocity limit. It consists of the product of the null vector $r^{\mu}$ connecting the emitting and absorbing vertices with an invariant expression proportional to $\left(1 / \mathbf{r}^{2}\right)$ in the zero velocity limit. The impulse is reminiscent of the Liénard-Wiechert potential but symmetrized between the source momentum $p$ and the absorber momentum $q$ :

$$
l^{\mu}=\alpha p \cdot q r^{\mu} / r \cdot p r \cdot q .
$$

We refer to Eq. (3) as a $p q$ impulse due to the $p \cdot q$ term in the numerator. Other possible impulses are discussed in Sec. V.

The null radius vector $r=y-x$ runs from the source point $x=x_{n}$ to the absorber point $y=x_{n+1}$. We choose $p_{n-2, n}$ as the source momentum $p=p_{i}$ and $p_{n-1, n+1}$ as the absorber momentum $q$. The roles and the labeling of the particles are interchanged for successive impulses, but the same mass is always associated with a specific particle trajectory (odd or even indices). The dimensionless coupling constant $\alpha$ is $\pm e^{2} / \hbar c \approx \pm 1 / 137$ for electrodynamics, but can be arbitrarily chosen. In the equal mass case, the asymptotic value of the denominator $r \cdot p r \cdot q \rightarrow r^{2} m^{2}$.

As written, $l^{\mu}$ is a "bare" impulse and does not necessarily give $\left(p_{f}\right)^{2}=m^{2}$, unless a correction factor $\eta$ is used to define the "physical" impulse

$$
k^{\mu}=\eta l^{\mu} .
$$

Requiring

$$
m^{2}=\left(p_{f}\right)^{2}=\left(p_{i}+k_{i}-\eta l_{f}\right)^{2},
$$

and noting that $k^{2}=l^{2}=0$, we get

$$
\eta=p_{i} \cdot k_{i} / l_{f} \cdot\left(p_{i}+k_{i}\right) .
$$

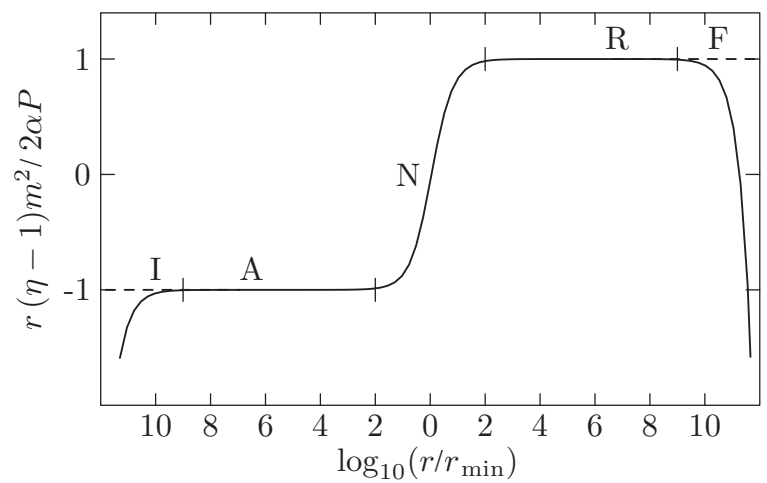

FIG. 2. Normalized $\eta-1$ for Eq. (3) with $P=0.3 m$ and equal masses. This plot was calculated with $\theta=90^{\circ}$, but does not, in fact, depend on $L$. The product $r(\eta-1)$ is constant far from the turning point, both while approaching (A) and while retreating (R). There is a transition in the region (N) when $r<100 r_{\min }$ and departures from constancy in the initial (I) and final (F) parts when $\eta_{o}=1$. With an appropriate estimate of $\eta_{o}$, (I) and (F) become extensions of (A) and (R) (dashed lines).

The factor $\eta$ is very close to 1 for large distances or for low energies. The product $r(\eta-1)$ is approximately constant far from closest approach $\left(r>100 r_{\min }\right)$. This is illustrated in Fig. 2 for $p q$ impulses (3). The bare impulse represents the asymptotic value and is useful for comparison to other theories. The deviation from (3) at finite distances is given by $\eta-1$.

There are, however, problems for attractive impulses $(\alpha<$ $0)$ at high energies or very small angular momenta when it is possible that $\eta<0$, which changes the interaction to repulsive. Similar behavior has been noted for a relativistic test particle in a static Coulomb field [2]. When $L<|\alpha|$, the distance of closest approach becomes negative, the particle is captured and it may spiral into the origin.

\section{COMPUTATIONAL DETAILS}

Numerical calculations of trajectories were performed in the center-of-momentum system with the coordinate origin at the center-of-energy. Because of the wide range of momenta and initial conditions explored, numerical calculations are a challenge. Machine precision (about 20 decimal digits) was insufficient, so the the multiple precision package GMP [3] was adopted. See the Appendix for more details.

The one-impulse-in-transit model is easily computable because the next interaction vertex can be found from the previous two vertices and the constant velocity of the absorbing particle. Using several impulses in transit would complicate this determination, perhaps requiring iterations and a protocol for splitting impulses. What is remarkable is how well the single impulse model succeeds.

Units are chosen so $\hbar=c=1$. For electrodynamics, the dimensionless coupling constant is $\alpha= \pm e^{2} / \hbar c \approx \pm 1 / 137$. Note that $\alpha$ is signed to treat both repulsive and attractive interactions. Our metric is such that $p^{\mu} p_{\mu}=E^{2}-\mathbf{p} \cdot \mathbf{p}=$ $m^{2} \geqslant 0$.

By choosing the center-of-momentum system with the origin at the center-of-energy $\overline{\mathbf{x}}=\sum E_{i} \mathbf{x}_{i} / \sum E_{i}$, the point of closest approach stays near the coordinate origin, regardless 
TABLE I. Scale and number of interaction vertices for electrons with $P=m_{e}=1$ at selected initial distances $R$ from the origin. At this velocity, most of the momentum transfer $(\approx 95 \%)$ occurs at the closest four vertices. The number of vertices increases as $\log R$ due to the increasing times for impulse transit; this number also scales roughly as $1 / v$.

\begin{tabular}{llc}
\hline \hline$R$ & \multicolumn{1}{c}{ Scale } & Vertices $(v=0.7 c)$ \\
\hline $10^{10}$ & 0.4 millimeters & 34 \\
$10^{20}$ & 0.6 Earth radius & 62 \\
$10^{30}$ & 4 light years & 90 \\
$10^{40}$ & 2 Hubble lengths & 120 \\
\hline \hline
\end{tabular}

of the initial separation. Moreover, the angular momentum of the system in these coordinates

$$
L^{\mu \nu}=\sum_{j} p_{j}^{\mu}\left(x_{j}-\bar{x}\right)^{\nu}-\left(x_{j}-\bar{x}\right)^{\mu} p_{j}^{\nu}
$$

has only the familiar spacelike components of $\mathbf{L}$. One must calculate $L^{\mu \nu}$ using positions at the same time and including the impulse in addition to the two particles.

Since the four-momentum of the null impulse goes to zero at large distances, $(\omega, \mathbf{k}) \rightarrow(0, \mathbf{0})$, we take $P$ as the magnitude of the asymptotic space momentum of either particle. In the case of unequal masses, we distinguish $E_{a}=\sqrt{P^{2}+m_{a}^{2}}$ and $E_{b}=\sqrt{P^{2}+m_{b}^{2}}$, whereas when $m_{a}=m_{b}$ we may simply write $E$ and $m$. Usually we choose $m$ to be the electron mass and set $m=1$; in the case of unequal masses, $m_{b}$ is usually set larger than $m_{a}$. Thus the unit length is the reduced Compton wavelength of the electron, $\hbar / m_{e} c$. Large initial distances $\left(R>10^{20}\right)$ were needed to provide suitably asymptotic values for scattering angles and total action (see Table I).

Since the particles and the impulse travel at known constant velocities, the transit time and the absorption point of an impulse can be determined. Let $\Delta t=\left(t^{\prime}-t\right)$ be the time interval for an impulse between its emission at $(t, \mathbf{x})=x_{4}$ by the particle having momentum $p_{i}=p_{2,4}$ to its absorption at point $\left(t^{\prime}, \mathbf{y}^{\prime}\right)=x_{5}$. The absorbing particle has had the momentum $q=q_{3,5}$ since emitting the previous impulse $k_{i}=$ $k_{3,4}$ at $(t-\delta t, \mathbf{y}-\mathbf{v} \delta t)=x_{3}$, where $\mathbf{v}=\mathbf{q} / E_{q}$. The equal-time separation of the particles at $t$ is $\mathbf{d}=\mathbf{y}-\mathbf{x}$ and the retarded radius is $\mathbf{r}=\mathbf{y}^{\prime}-\mathbf{x}=\mathbf{d}+\mathbf{v} \Delta t$. Requiring a null interval $\mathbf{r}^{2}=$ $c^{2}(\Delta t)^{2}$ for the transit of the impulse $k_{4,5}$ gives the equation $\left(c^{2}-v^{2}\right)(\Delta t)^{2}-2 \mathbf{d} \cdot \mathbf{v}(\Delta t)-\mathbf{d}^{2}=0$. This has solutions

$$
\Delta t=(\gamma / c)^{2}\left[\mathbf{d} \cdot \mathbf{v} \pm \sqrt{(\mathbf{d} \cdot \mathbf{v})^{2}+c^{2} \mathbf{d}^{2} / \gamma^{2}}\right]
$$

where $\gamma^{2}=c^{2} /\left(c^{2}-v^{2}\right)=E^{2} / m^{2} c^{4}$ and velocities are expressed as $\beta=\mathbf{v} / c=\mathbf{p} c / E$. The solution with the positive sign is the future $\Delta t$ for $k_{4,5}$ and the solution with the negative sign is the past $\delta t$ for $k_{3,4}$.

One difficulty with an impulse exchange dynamics is initialization. An incoming impulse is needed for the first interaction vertex. The approach adopted is to take a large starting separation and project the motion of one particle further backward in time to find the vertex at which it would emit an impulse to the first calculated interaction vertex. That impulse is calculated assuming a zero incoming impulse. The process is iterated to adjust the momenta $p, q$, and $k$ at the starting separation so the total initial four-momentum of the particles and impulse equals the asymptotic $(2 E, \mathbf{0})$. To be more explicit, I will give a rough sketch of the algorithm for the case of equal masses $m$, asymptotic particle momenta $P$, total angular momentum $L$, and initial particle separation $2 R$ at $t_{1}=$ $-|R E / P|$. Take the initial particle momenta to be $Q=P$.

(i) Define the starting momenta and positions of the two particles, one (a) with momentum $p=\left(E_{Q}, Q, 0,0\right)$ at $x_{1}=\left(t_{1},-R,-b / 2,0\right)$ and the other (b) with momentum $q=\left(E_{Q},-Q, 0,0\right)$ at $y_{1}=\left(t_{1}, R, b / 2,0\right)$, where $b=L / Q$.

(ii) Project the position of (a) back in time to $x_{0}$ so a null impulse emitted will reach $y_{1}$ at $t_{1}$. Calculate the corresponding null impulse $k=k_{0,1}$. One cannot calculate $\eta_{o}$ because there is no previous impulse. Either set $\eta_{o}=1$ or, preferably, estimate $\eta_{o}$ as described below.

(iii) Recalculate $\mathbf{p}=-\mathbf{q}-\mathbf{k}$ so the space momentum of the system is zero, and put $p$ on the mass shell with $E_{p}=\sqrt{\mathbf{p}^{2}+m^{2}}$. In the resulting system, the particles and the impulse are on the mass shell, but the total energy may differ from the asymptotic $2 E$.

(iv) Solve $\sqrt{(-\mathbf{q}-\mathbf{k})^{2}+m^{2}}+\sqrt{\mathbf{q}^{2}+m^{2}}+|\mathbf{k}|=2 E$ for $Q=|\mathbf{q}|$. Then repeat steps (i) through (iv), recalculating $p$, $q$, and $k$, until the total energy is sufficiently close to $2 E$.

(v) When the iteration converges, successively interchange the roles of $p$ and $q$ and proceed to calculate the trajectory.

(vi) When the particles are traveling outward and reach a separation greater than $2 R$, evaluate the scattering angle.

If an appropriate initial $\eta_{o} \neq 1$ is used, the trajectory evolves as if it had started in the infinite past [see Fig. 2, regions (I) and (F) and Table II]. The form of the initial $\eta_{o}$ correction is suggested by the the value of $r(\eta-1) / \alpha$ at distances far from the origin. Finding the exact expression requires some experimentation. For equal masses, $\eta_{o}-1=2 \alpha E / m^{2} r$, where $r$ is the distance traveled by the null impulse. See Sec. V for more discussion.

A simplified implementation of this algorithm has been written in MAXIMA [4] and is furnished as Supplemental Material [5].

\section{TRAJECTORIES AND CROSS SECTIONS}

Classical trajectories are smooth curves as seen in Fig. 3. In contrast, impulse trajectories involve straight line travel between interaction vertices. Explicitly treating two interacting particles, rather than reducing the problem to a central

TABLE II. The $1 / r$ dependence of $\eta-1$ and $\eta_{o}-1$ for regions A and I of Fig. 2. Two types of impulses and two vertex excitations are given for particles with equal masses. With the apppropriate choice of $\eta_{o}$, the constant value of region A extends to region I. The distance traveled by the photon is $r$, and the distance from the emission vertex to the origin is $r_{e}=(E+P) r / 2 E$ for a particle approaching the origin.

\begin{tabular}{lccc}
\hline \hline Eq. & Excited & $|\eta-1|$ & $\eta_{o}-1$ \\
\hline$(3)$ & No & $2 \alpha P / m^{2} r$ & $2 \alpha E / m^{2} r$ \\
$(3)$ & Yes & $\alpha / 2 E r_{e}$ & $\alpha / 2 E r_{e}$ \\
$(18)$ & No & $\alpha / E r_{e}$ & 0 \\
$(18)$ & Yes & 0 & 0 \\
\hline \hline
\end{tabular}




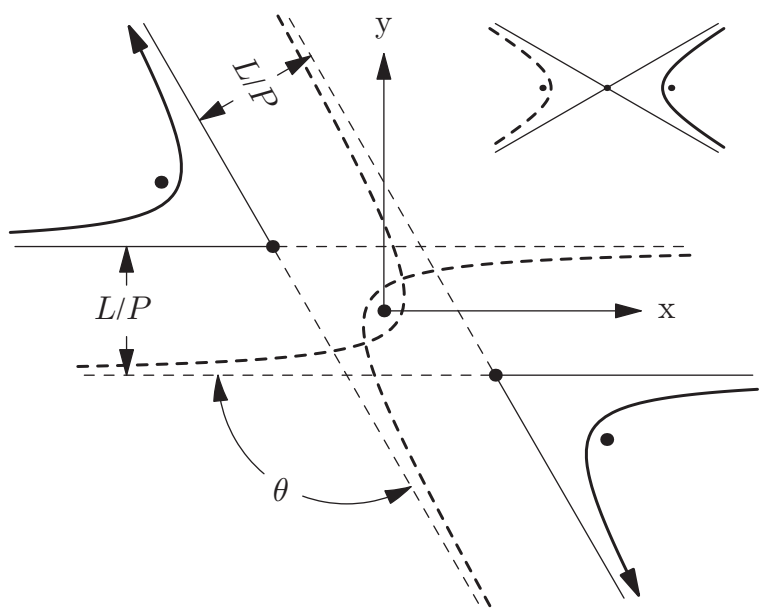

FIG. 3. Classical hyperbolic trajectories. The two branch hyperbola (shown in the upper right corner) depicting single particle, central force trajectories is doubled and offset when two particles are considered. Repulsive and attractive interactions are depicted by solid and dashed trajectories, respectively. The scattering angle $\theta$ is $120^{\circ}$. The equal mass case is shown. For unequal masses, the relative scale of the paths changes.

force calculation, requires two sets of hyperbolae with offset asymptotes to accommodate their mutual angular momentum. A sampling of impulse trajectories for equal mass particles interacting with a $p q$ impulse (3) is given in Fig. 4. To include all vertices, a reciprocal plot was used. This emphasizes changes of direction near the turning point and drastically suppresses the display of the nearly straight line travel in the asymptotic regions.

In the impulse case, the motion is qualitatively hyperbolic with almost straight asymptotes and most of the turning occurring within 16 times the distance of closest approach. For larger momenta, $(v>0.1 c)$, the fewer impulses near the turning point no longer blend visually into a smooth curve.
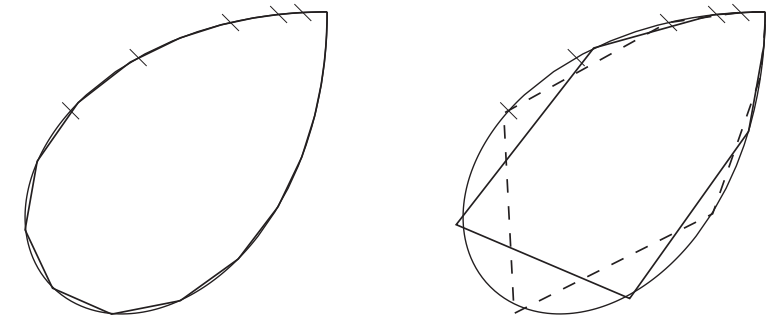

$$
\text { (a) } v=0.1 c, 1 \text { traj. }
$$

(b) $v=0.33 c, 2$ traj.

For $\eta$-corrected impulses in the center-of-momentum system, there is a simple relation between the asymptotic scattering angle $\theta$ and the angular momentum $L$ for fixed $P, m_{a}, m_{b}$, and $\alpha$ :

$$
L \tan (\theta / 2)=L_{90}=\alpha p \cdot q / P\left(E_{a}+E_{b}\right),
$$

where $P$ is the asymptotic momentum of either particle, $E_{a}=$ $\sqrt{P^{2}+m_{a}^{2}}$, and $E_{b}=\sqrt{P^{2}+m_{b}^{2}}$. The asymptotic initial four-vectors in $p \cdot q$ are $p=\left(E_{a}, \mathbf{P}\right)$ and $q=\left(E_{b},-\mathbf{P}\right)$. I have found it useful to denote the right-hand side of Eq. (8) as $L_{90}$, the angular momentum for $90^{\circ}$ scattering.

In the low velocity limit, (8) reduces to the familar classical result [6],

$$
L \tan (\theta / 2)=\alpha m_{a} m_{b} / P\left(m_{a}+m_{b}\right) .
$$

Only a finite number of photons is needed to calculate a scattering angle to any desired accuracy. The asymptotic relation (8) was inferred from numerical trends and verified computationally. Although the varying nearest approaches seen in Fig. 4 at high energies may suggest that small changes in the emission times of the initial photon could lead to significantly different scattering angles, the convergence of the calculated $\tan (\theta / 2)$ to the asymptotic value is rapid. For equal masses, the difference decreases as $1 / R^{2}$ and, for unequal masses, as $1 / R$ (where $2 R$ is the initial and final separation). Thus, at a sufficiently large starting distance, changes in the initial distance (or time) result only in small and predictable changes in the calculated scattering angle.

For $\alpha<0, L$ is the same as for $|\alpha|$. However, for nearly head-on collisions, impulses can become so negative that the invariant $m^{* 2}=\left(p_{i}+k_{i}\right)^{2}<0$ and $\eta$ changes sign, changing the interaction to repulsive. In the range $0.001 m<P<$ $1000 \mathrm{~m}$, this happens for $L<|\alpha| E / \mathrm{m}$. The bound is imprecise because the details of closest approach depend on the initial separation $2 R$. One can introduce an alternative scaling of the impulse, $m^{2} /\left(m^{2}-m^{* 2}\right)$, to avoid the sign change, but this results in energy loss and capture into orbit. Similar behavior,

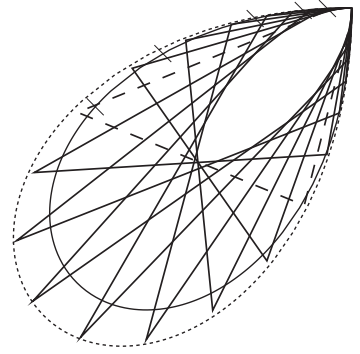

(c) $v=0.66 c, 8$ traj.

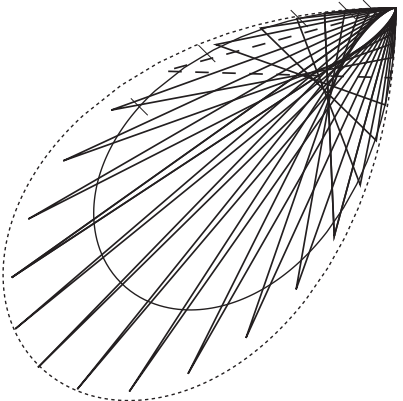

(d) $v=0.9 c, 16$ traj.

FIG. 4. Reciprocal vertex positions for impulse trajectories with equal mass particles, repulsive interaction, $90^{\circ}$ scattering. For a vertex at $(x, y)$, the point $\left(x r_{\min } / r^{2}, y r_{\min } / r^{2}\right)$ is plotted, where $r^{2}=x^{2}+y^{2}$ and $r_{\min }=\left(\alpha E+\sqrt{\alpha^{2} m^{2}+4 P^{2} L^{2}}\right) / 4 P^{2}$ for a static potential $\alpha / 2 r$. The connecting chords are straight lines in the original space and are depicted as straight lines in this inverse mapping, whereas they would, in fact, be arcs of circles. The "teardrop" shaped solid line shows the inverse of a hyperbola with semiaxes matching the asymptotes of the actual trajectories. The ticks on the upper boundary correspond to $r / r_{\min }=\sqrt{2}, 2,4,8$, and 16 . For $v>0.1 c$, ensembles of trajectories are shown, generated by varying the initial separation of the particles. One representative trajectory is distinguished by a dashed line. The points of closest approach are nearer than the reference hyperbola and have an envelope (outer dotted line). As $v$ increases, there are fewer vertices on a trajectory and fewer vertices near the turning point. 
when $\alpha<0$, has been noted for a classical relativistic test particle in a static Coulomb field [2].

Impulse dynamics is constructed and calculated using the formalism of special relativity; it is relativistic. The low velocity limit gives the familiar motion of Newtonian particles in a central potential. The remaining point at issue is whether and when the impulse reproduces the interactions seen at relativistic velocities, either in quantum scattering or in classical orbits. We will show how well the $p q$ impulse Eq. (3) succeeds and see that another candidate, the $m M$ impulse Eq. (18), does not. To facilitate comparisons, we convert Eq. (8) into a differential cross section.

$$
\left.\frac{d \sigma}{d \Omega}\right|_{\mathrm{ID}}=\frac{\alpha^{2}}{4\left(E_{a}+E_{b}\right)^{2} P^{4}} \frac{(p \cdot q)^{2}}{\sin ^{4}(\theta / 2)} .
$$

This is a relativistic generalization of the Rutherford cross section. Note that $\left(E_{a}+E_{b}\right)^{2}$ can be written $(p+q)^{2}$.

Quantum differential cross sections for the equal mass case have been simplified to apply to distinguishable particles by removing the interference terms and the second scattering peak arising from the identical particle symmetrization. We introduce asymptotic final four-vectors corresponding to the initial $p$ and $q$ above: $p^{\prime}=\left(E, \mathbf{P}^{\prime}\right)$ and $q^{\prime}=\left(E,-\mathbf{P}^{\prime}\right)$, where $p^{\prime} \cdot p=E^{2}-P^{2} \cos \theta$ and $\left(p^{\prime}-p\right)^{2}=-2 P^{2}(1-\cos \theta)=$ $-4 P^{2} \sin ^{2}(\theta / 2)$. The Møller cross section [7] becomes

$$
\left.\frac{d \sigma}{d \Omega}\right|_{\mathrm{QED}}=\frac{\alpha^{2}}{2 E^{2}}\left\{\frac{(p \cdot q)^{2}+\left(p \cdot q^{\prime}\right)^{2}+2 m^{2}\left(p \cdot q^{\prime}-p \cdot q\right)}{\left[\left(p^{\prime}-p\right)^{2}\right]^{2}}\right\} .
$$

Scalar electrodynamics gives

$$
\left.\frac{d \sigma}{d \Omega}\right|_{\mathrm{SED}}=\frac{\alpha^{2}}{4 E^{2}}\left[\frac{p \cdot q+p \cdot q^{\prime}}{\left(p^{\prime}-p\right)^{2}}\right]^{2} .
$$

The ratio of the Møller cross section to the impulse cross section is

$$
1+\left(p^{\prime}-p\right)^{2} E^{2} /(p \cdot q)^{2}+\left(p^{\prime}-p\right)^{4} / 8(p \cdot q)^{2},
$$

and the ratio for scalar electrodynamics is

$$
1+\left(p^{\prime}-p\right)^{2} / 2 p \cdot q+\left(p^{\prime}-p\right)^{4} / 16(p \cdot q)^{2} .
$$

These ratios are almost unity when the distance of closest approach is relatively large. This happens for small angles (at any velocity) and for small velocities (at any angle), defining what we will call the classical interaction region. All three expressions, Eqs. (10), (11), and (12), share the same leading term, proportional to $(p \cdot q)^{2}$, which accounts for low-angle relativistic scattering and for nonrelativistic scattering. The correction terms, proportional to powers of $\left(p^{\prime}-p\right)^{2}$, are, at least in part, of quantum origin, since they differ between the scalar and spinor (QED) theories. Quantum effects are important $(>1 \%)$ only for relativistic velocities when distances of closest approach can be less than the Compton wavelength of the particle, in what we will call the quantum interaction region. The classical interaction region extends to well below the Bohr radius for electrons and to nuclear dimensions for protons.

The correction terms are unimportant $(<1 \%)$ for forward scattering $[\sin (\theta / 2)<0.1]$ and for small velocities $(v<0.1)$. The corrections can be much larger when the distance of closest approach is less than the Compton wavelength of the particle. For $90^{\circ}$ scattering, the largest disagreement occurs in the transition between nonrelativistic and ultrarelativistic motion for momenta $0.1 m<P<10 m$.

In the classical interaction region, the scattering of $p q$ impulses agrees with quantum scattering. At closer approaches, differences arise due to quantum effects such as spin and interference. The form of the impulse is important. A second candidate impulse, the $m M$ impulse Eq. (18), does not agree at high energies because its leading term is proportional to $\left(m_{a} m_{b}\right)^{2}$ rather than to $(p \cdot q)^{2}$.

A more artificial comparison of scattering is with a relativistic test particle in a central potential [2,8]. Calculation of orbits or trajectories of a relativistic test particle in a static potential seems easy because one can apparently ignore the problems of recoil and retardation. The central mass is assumed to be infinite so it will not move and thus retardation and recoil need not be considered. In contrast, scattering in impulse dynamics handles retardation and recoil correctly; one can then take the limit of one mass going to infinity.

In the limit $m_{a}=m$ and $m_{b} \rightarrow \infty$, (10) becomes

$$
\left.\frac{d \sigma}{d \Omega}\right|_{\mathrm{ID}} \rightarrow \frac{\alpha^{2} E^{2}}{4 P^{4} \sin ^{4}(\theta / 2)},
$$

which can be compared to the Mott cross section

$$
\left.\frac{d \sigma}{d \Omega}\right|_{\text {Mott }}=\frac{\alpha^{2} E^{2}\left[1-v^{2} \sin ^{2}(\theta / 2)\right]}{4 P^{4} \sin ^{4}(\theta / 2)},
$$

and to a cross section derived from the analytic solutions for a classical relativistic test particle in a static central potential [8]. No simple formula comparable to the above is available, but using $d \sigma / d \Omega=(b / \sin \theta) /|d \theta / d b|=L /\left(\sin \theta P^{2}|d \theta / d L|\right)$ and $\lambda=\sqrt{\left|L^{2}-\alpha^{2}\right|}$,

$$
\frac{d \theta}{d L}=\frac{2 \alpha}{\lambda}\left[\frac{\alpha \tan ^{-1}(v \lambda / \alpha)}{\lambda}-\frac{v L^{2}}{v^{2} L^{2}+\alpha^{2}\left(1-v^{2}\right)}\right] .
$$

This holds for $L>\alpha>0$; for $\alpha>L>0$, replace $\tan ^{-1}$ () with $\tanh ^{-1}($ ). A graphical comparison of (15), (16), (17) is given in the Supplemental Material [5].

For nonforward scattering, the angular momentum for scattering to a given angle is larger for impulses than for the test particle, so the impulse cross sections are larger. The difference is important only for small impact parameters in the quantum interaction region. The Mott expression (16) differs from (15) by a factor $\left[1-v^{2} \sin ^{2}(\theta / 2)\right]$. This factor, a third example of quantum correction terms, is $\left[1-v^{2}\right]$ in the backward direction and greater than $\left[1-\sin ^{2}(\theta / 2]\right)$ for forward scattering. The regions of agreement are similar to those found in the equal mass case.

\section{OTHER IMPULSES}

As mentioned above, we have considered other relativistic impulses. All of them reduce to a Coulomb-like interaction in the zero velocity limit but differ in their effective coupling as velocities increase. Replacing $p \cdot q$ in the numerator of Eq. (3) with $m_{a} m_{b}$,

$$
l^{\mu}=\alpha m_{a} m_{b} r^{\mu} / r \cdot p r \cdot q .
$$


We refer to Eq. (18) as an $m M$ impulse. The numerator term $m_{a} m_{b}$ gives a smaller effective coupling constant for high energies than is the case for Eq. (3).

There is also a choice of either $p_{i}$ or $p_{i}+k_{i}$ as the source momentum $p$; we will call these "unexcited" and "excited" vertices, respectively. Our original choice for the $m M$ impulse Eq. (18) was to use an "excited" emission vertex $p=p_{\text {vertex }}=$ $p_{n-2, n}+k_{n-1, n}$ because it gave $\left(p_{f}\right)^{2}=m^{2}$ numerically (i.e., $\eta=1)$ and seemed to lead to simpler results for the angular dependence in scattering. With either choice (excited or unexcited) for the source vertex $p$, the corresponding $\eta$ correction changes $l^{\mu}$ to the (numerically) same physical impulse $k^{\mu}$. It does not matter whether the emission vertex is excited. Initial $\eta_{o}-1$ values are given in Table II . The case of unequal masses is similar, but formulas for the exact values of $\eta_{o}-1$ have not been determined. If needed, one can iteratively determine the multiplier, starting from the equal-mass case.

For $m M$ impulses Eq. (18), we infer numerically

$$
L \tan (\theta / 2)=\alpha m_{a} m_{b} / P\left(E_{a}+E_{b}\right) .
$$

This corresponds to Eq. (8) for $p q$ impulses (3). It also reduces to (9) when $v \rightarrow 0$ but has a smaller value than Eq. (8) when $m_{a} m_{b}<p \cdot q$. As $v \rightarrow 1, L_{90} \rightarrow \alpha$ for Eq. (3), but for Eq. (18), $L_{90}$ continues to decrease.

We also tried substituting $[r \cdot(p+q)]^{2}$ for the previous denominator $r \cdot p r \cdot q$ :

$$
l^{\mu}=\alpha(p+q) \cdot(p+q) r^{\mu} /[r \cdot(p+q)]^{2} .
$$

Equation (20) appears to be more like the classical Coulomb case, since $r \cdot(p+q) \approx r E_{\text {total }}$ in the center-of-momentum system. These other Coulomb-like impulses also have constant regions $(A, R)$ like those seen in Fig. 2 but differ in the near region $(\mathrm{N})$. Equations (20) and (18) give the same physical impulse once the corresponding $\eta$ corrections are applied.

Thus, there are only two families of effective impulses, equivalent to either Eq. (18) or Eq. (3). Since the required $|\eta-1|$ is considerably larger for Eq. (20), we conclude that the Liénard-Wiechert-like denominator is more descriptive of the physics.

One referee has pointed out that keeping particles on the mass shell with the $\eta$ correction makes only the choice of the initial photon magnitude important; subsequent to that, it suffices that $k^{\mu}$ is collinear with $r^{\mu}$ and that both are null fourvectors. This succinctly explains my numerical observations of two families of effective impulses.

As discussed in Sec. IV, the choice Eq. (3) for impulse electrodynamics is in broad agreement with relativistic Møller and Mott cross sections [7].

\section{CONNECTIONS TO CLASSICAL ELECTRODYNAMICS}

\section{A. Magnetism}

The $p q$ impulse Eq. (3) was constructed to give the dominant Coulomb interaction in the low-velocity limit. It is not immediately obvious that there are components that can be identified with magnetic forces. One motivation for the $p q$ impulse was the $-\mathbf{p} \cdot \mathbf{q}$ correction to the radial impulse that comes "free" from the four-vector dot product. Due to a plausible similarity of $k^{\mu}$ to the vector potential $A^{\mu}$, considerable effort was spent trying to add terms proportional to $p$ and $q$ to supply the $\mathbf{u}(\mathbf{v} \cdot \mathbf{r}) / r^{3}$ term of the classical magnetic force but to no avail.

Finally I realized that the classical expressions are stated in a Galilean frame with an instantaneous radius vector $\mathbf{r}_{\mathbf{o}}$ rather than a retarded one. Moreover, the relativistic LiénardWiechert forces can also be so stated, differing from the classical forces only by a multiplying factor [9]

$$
G\left(\mathbf{u}, \mathbf{r}_{\mathbf{o}}\right)=\gamma /\left[1+\left(\gamma \mathbf{u} \cdot \hat{\mathbf{r}}_{\mathbf{o}}\right)^{2}\right]^{3 / 2} .
$$

As $u \rightarrow 1, G$ narrows and peaks perpendicular to the direction of travel, modeling transverse photons; retardation modifies the character of peaking in the case of impulses.

Since we do not have fields in impulse dynamics, we must compare average impulse forces to Liénard-Wiechert forces. To make our comparisons explicit, we take $y=x_{4}$ in Fig. 1 as our "field" point and introduce a point $x$ on the line between $x_{3}$ and $x_{5}$ with the same time coordinate as $y_{4}$ as our equal time source point. Then $r_{o}=y-x$ and $\Delta t$ is the time component of $x_{5}-x_{3}$. The source velocity $\mathbf{u}$ corresponds to $p_{3,5}$ and we consider an arbitrary velocity $\mathbf{v}$ for the field point rather than one corresponding to the momentum $q_{2,4}$ or $q_{4,6}$ on a trajectory. The average impulse force is

$$
\mathbf{F}_{I}=\left(\mathbf{k}_{3,4}-\mathbf{k}_{4,5}\right) / \Delta t
$$

and the Liénard-Wiechert force is

$$
\mathbf{F}_{E}+\mathbf{F}_{B}=\alpha G\left[\mathbf{r}_{\mathbf{o}}+\mathbf{v} \times\left(\mathbf{u} \times \mathbf{r}_{\mathbf{o}}\right)\right] / r_{o}^{3} .
$$

Naively one might expect a stationary charge of opposite magnitude would cancel the Coulomb force $\mathbf{F}_{E}$ of a moving charge and leave a net magnetic force. Because of relativistic effects in the factor $G$, this happens only to order $u^{2}$, of the same order of magnitude $(u v)$ as the magnetic force. Because of the symmetry between $p$ and $q$ in the impulses, we find

$$
\left|\mathbf{F}_{I}-\mathbf{F}_{E}-\mathbf{F}_{B}\right| r_{o}^{2} \approx \alpha \max \left(u^{2}, v^{2}\right)
$$

which effectively masks the radial magnetic force.

Although I could not verify the radial magnetic force, the components of $\mathbf{F}_{I}$ and $\mathbf{F}_{B}$ perpendicular to $\mathbf{r}_{\mathbf{o}}$ do agree very well:

$$
\left|\mathbf{r}_{\mathbf{o}} \times\left(\mathbf{F}_{I}-\mathbf{F}_{B}\right)\right| r_{o} \approx \alpha u v \max \left(u^{2}, v^{2}\right) .
$$

Typical geometries with $\mathbf{v} \cdot \mathbf{r}_{\mathbf{o}} \neq 0$ have the perpendicular magnetic force smaller than $\mathbf{F}_{B}$, but it is possible to choose $\mathbf{v} \perp \mathbf{r}_{\mathbf{o}}$, making the full $\mathbf{F}_{B}$ nonradial while maintaining this agreement. For laboratory velocities of a few centimeters per second (fusing currents in copper wires have $u \approx v \approx 10^{-10} c$ ), this happens to an accuracy of $10^{-20}$. Useful agreement continues until $u \approx v \approx 10^{-2} c$ ), when the effects of retardation and discretization influence $\mathbf{F}_{I}$ in ways that differ from the peaking exhibited by $G$. In the comparison of the magnetic component of inpulse forces, there is good agreement for both types of impulses Eqs. (3) and (18) at low velocities; differences emerge at high velocities.

A better theory of neutral conductors is needed. The discrepancy noted in Eq. (24) also occurs with the conventional Coulomb force on a stationary charge due to the apparently neutral combination of a moving charge and a stationary 
counter charge of opposite sign. This is because $G \neq 1$ for the moving charge. The lack of cancellation is small $\left(\approx u^{2}\right)$, less than one electron per Coulomb of charge.

The purpose of this subsection was to show that classical (laboratory) magnetism is included implicitly in retarded impulses. At relativistic velocities, the magnetic force grows to match the radial force in magnitude and both are strongly modified by the factor $G$. It can be questioned whether Liénard-Wiechert potentials or forces suffice to describe extreme relativistic interactions, since they depend on projecting straight line trajectories backward to estimate retardation. Our discussion of relativistic cross sections in Sec. IV shows that the $p q$ impulse adequately describes classical interactions at high velocities and the predominance of forward scattering noted there does indicate that the Liénard-Wiechert forces should perform well.

We conclude that retardation (and anticipation) effects seen from the vantage point of an instantaneous radius vector account for classical magnetic forces. This is consistent with a magnetic field arising from a Lorentz transformation of a static Coulomb field.

\section{B. Radiation reaction and radiation}

Impulse dynamics already has a mechanism for radiation reaction. Conservation of four-momentum at a vertex serves to transfer energy and momentum between particles and radiation in the same way that it handles the impulse exchanges which describe interparticle interactions. A scalar null impulse corresponds to a radiation photon as in the classical theory of the Compton effect; it is a point entity so the transfer is immediate and local rather than being modelled by an integrated flux at infinity as in classical electrodyanmics.

A rudimentary theory of radiation from low-energy particles can be developed by supposing that the probability of radiating an impulse from a vertex $n$ is proportional to an excitation

$$
X_{n}(\Delta q)=\sqrt{-\left(k_{i}-k_{f}\right)_{n}^{2}} \approx X_{n}^{*}=\left[\left(p_{i}+k_{i}\right)_{n}^{2}-m^{2}\right] / m
$$

and that the spectrum does not depend on the energy of the emitted impulse. The excitation $X(\Delta q)$ is general, while $X^{*}$ is defined only for $\alpha>0$. For low energies, $X^{*}$ and $X(\Delta q)$ are numerically close.

This gives an incremental energy loss

$$
\left.\Delta W_{n} \text { [impulse }\right]=-X_{n} \int_{0}^{\omega_{\max }} \omega d \omega / m^{2} .
$$

The maximum energy $\omega_{\max }$ of a radiated impulse $k$ is restricted by the requirement that sufficient excitation remains after radiation to continue the exchange of impulses which describe the interaction between the charges. This maximum depends on the vertex. It is largest near the turning point or with small $L$. It falls off at large distances and large impact parameters.

The energy loss computed from (27) along a nonrelativistic hyperbolic trajectory is proportional to the loss computed from the Larmor radiation damping formula, based on average accelerations. Note that both the impulse and the Larmor energy losses were computed by assuming that no actual radiation occurred and that the elastic collision trajectory was maintained by external means. Also, it is problematic to calculate the trajectories of relativistic charges in classical electrodynamics. I expect to be able to address these issues by comparing an ensemble of impulse trajectories including radiative losses to the first-order quantum electrodynamics bremstrahlung cross section [10].

\section{QUANTUM MECHANICS}

\section{A. Probabilities and couplings}

Impulse dynamics gives broken line trajectories, continuous but not differentiable at the interaction vertices. Ensembles of trajectories with different initial positions, but the same $L$ and asymptotic $P$, give a relationship between $L$ and the scattering angle $\theta$. There is no potential originating from a single particle as in the classical case, only photonlike impulses in transit. In the Newtonian limit, $(v \rightarrow 0)$, forces corresponding to classical potentials can be recovered from the difference between absorbed and emitted impulses.

Taking impulses as the underlying mechanics, classical potentials are at most a statistical property of an ensemble of impulses and may not be recoverable when retardation is relevant. From this point of view, potentials or forces do not determine the classical interaction of particles but rather describe the average local behavior of an ensemble of particles interacting via impulses. If potentials are statistical, a quantum mechanics based on potentials is intrinsically statistical, relative to predictions of particle motion described by impulse exchange or to seemingly deterministic classical calculations.

Classical impulses are scalars without the vector polarization of conventional photons. Since both are massless, I tend to identify impulses with photons. In standard quantum electodynamics, two spinor particles are coupled to a single vector photon (either absorbed or emitted) at an interaction vertex. In impulse dynamics, with the continual exchange of impulses, interaction vertices would couple four quanta (two particles and two impulses). This suggests that interaction impulses will be realized with spinors carrying the momemtum exchanges over finite distances and not by vectors describing asymptotic momentum transfers. The conventional vector field would be the difference between pairs of incoming and outgoing impulses, summed over the entire trajectory.

Radiation is still a candidate for a vector coming in from infinity or going out to infinity. For consistency, even this radiation might be treated as a spinor; the vector nature of photons arises from the vector nature of the electromagnetic field which impulse dynamics does not use.

\section{B. Action and phase shifts}

One approach to quantum scattering is to compare the phase shift between interacting particles and free particles with the same asymptotic states. The Coulomb case is made difficult by a logarithmic divergence of this phase difference due to the long range of the interaction [11].

In the nonrelativistic expansion of a free plane wave by spherical waves, the asymptotic phase is $\operatorname{Pr}+\pi L / 2$. The difference between the asymptotic phase of a nonrelativistic 
Coulomb wave function and a free plane wave is

$$
-\Lambda \ln 2 \operatorname{Pr}+\delta_{L}[\mathrm{nr}] .
$$

Conventionally, $\Lambda$ is written as $n, P$ as $k, L$ is restricted to integer values, and

$$
\delta_{L}[\mathrm{nr}]=\arg \Gamma(L+1+i n) .
$$

In quantum mechanics, the phase of a scattering amplitude or wave function is proportional to the classical action. Instead of arguing, as Feynman did [12], that interference restricts the contributions to possible paths near the classical path, we simply compute the action difference along a classical impulse trajectory

$$
S_{L}=\sum_{n \rightarrow n+2} S_{n}=\sum_{\text {even or odd }}^{\text {vertices }}\left(p^{\mu} \Delta x_{\mu}-m \Delta \tau_{\text {free }}\right)_{n} .
$$

For a single trajectory, the sum is over every other $n$, even or odd. The action of the interacting particle between interaction vertices, $\left(p^{\mu} \Delta x_{\mu}\right)_{n}=m^{2} \Delta t_{n} / E_{n}$, differs from $m \Delta \tau_{\text {free }}=m^{2} \Delta t_{n} / E$, the action of a free particle with mass $m$ and constant momentum $P$. Subtraction of $\sum m \Delta \tau_{\text {free }}$ removes the large linear term in the action which corresponds to the asymptotic phase $\mathrm{Pr}$ in the nonrelativistic case.

The individual terms in Eq. (30),

$$
S_{n}=p_{n, n+2}^{\mu}\left(x_{n+2}-x_{n}\right)_{\mu}-m^{2}\left(t_{n+2}-t_{n}\right) / E,
$$

are numerically constant on a single trajectory. They depend on $P, m$, and $\alpha$ but not on $L$. This can be seen as a continuum analog of the Bohr orbital quantum condition. We find numerically that $S_{n}\left(m_{a}\right)=2 L_{90} P / E_{a}$ and $S_{n}\left(m_{b}\right)=$ $2 L_{90} P / E_{b}$. In the equal mass case for a $p q$ impulse Eq. (3), $S_{n}=\alpha\left(1+v^{2}\right)$, where $v=P / E$.

Like the Coulomb phase shift, this net action Eq. (30) exhibits a logarithmic divergence, $S \rightarrow \Lambda \ln (r)$, which does not depend on $L$. The coefficient $\Lambda$ can be determined numerically from $S_{n} \rightarrow \Lambda \ln \left(r_{n} / r_{n+2}\right)$. In this section, $r$ is the distance from a vertex to the origin, not the distance traveled by an impulse between vertices. Assuming $L=0$, one can derive the limit $r_{n+2} / r_{n} \rightarrow(1+v)(1+u) /(1-v)(1-u)$, where $v=P / E_{a}$ and $u=P / E_{b}$. These sign choices hold when the particles are moving away from each other. For $m_{a}=m_{b}$, this gives $\Lambda=v L_{90} / \ln [(1+v) /(1-v)]$.

However, the calculated action $S_{L}$ depends on the final radial distance $r_{f}$, which changes with $L$, and on the initial distance $r_{i}$. To remove the asymptotic dependence on $r_{i}$ and $r_{f}$, we define $\tilde{S}_{L}=S_{L}-\Lambda \ln \left(r_{i} r_{f}\right)$. For $\alpha>0$, the difference between phase shifts for $L>0$ and $L=0$ is numerically found to be

$$
\delta_{\rho}=\tilde{S}_{L}-\tilde{S}_{0}=-\Lambda \ln \left(1+\rho^{2}\right),
$$

where $\rho=L / L_{90}=\cot (\theta / 2)$ and $L_{90}$ corresponds to $90^{\circ}$ scattering. The calculations show that changing the sign of $\alpha$ simply changes the signs of $\Lambda$ and $\tilde{S}_{L}$. Another reference point must be chosen for $\alpha<0$, since $\tilde{S}_{0}$ is not defined.

With these results, an $S$ matrix for scalar interactions should be calculable. An extension to spin and a comparison to quantum field theoretic results is beyond the scope of this paper.

\section{SUMMARY}

\section{A. Results and open questions}

We have explored an alternative model for the classical electrodynamic interaction of elementary charged particles. The theory satisfies special relativity and conserves fourmomentum by construction. Impulses replace forces. Highprecision numerical algebra replaces retarded differential equations. Since we consider only interactions between charges and do not use fields, the question of self-energies does not arise. Trajectories are calculated without having to deal with runaway solutions. The nonrelativistic relation between angular momentum and scattering angle is extended to the relativistic domain. We argue that photonlike impulses include both radial Coulomb forces and transverse magnetic forces. Classical potentials may in fact be statistical and descriptive only of the static limit. Hence a quantum mechanics based on them is "born" statistical. We suggest a net action to describe relativistic phase shifts.

Impulse dynamics is incomplete. The theory of radiation is rudimentary. How does one do quantum mechanics or quantum field theory without potentials? What about other types of impulses? For instance, $-|\alpha| r^{\mu}$ might describe quark interactions. Can one calculate trajectories for three or more interacting particles when the extrapolation of future position is not straightforward or perhaps only possible by successive approximation?

\section{B. Revisiting Newton's laws}

Newton's laws survive in an instructively modified, but strengthened, form. Since interactions occur only at separated vertices, particles travel with their free velocities almost all the time; they are not subject to forces acting continuously. Conservation of energy and momentum at interaction vertices encompasses the other two laws (force and reaction). The four-momentum of a particle changes abruptly as the result of absorption or emission of an impulse. The change is in the direction of the retarded (or advanced) radius. Radiation reaction is the conservation of four-momentum at emission; the emitting particle loses exactly what the impulse carries off. By focusing on the flow of energy-momentum, both of the particles and of the impulses, an equal and opposite reaction occurs locally at the vertex. The problems with magnetic forces and forces propagating at finite velocities disappear when the impulses are considered in addition to the particles alone.

\section{ACKNOWLEDGMENTS}

The germ of the idea for eliminating self-energies by considering only interparticle interactions was suggested in a seminar given by M. Sachs and M. Schwebel [13]. I thank R. Swanson, S. A. Fulling, R. Allen, W. Saslow, and a referee for comments and questions that have markedly improved the manuscript.

\section{APPENDIX: NUMERICAL ACCURACY AND CONSTRAINTS (INFERRING RELATIONSHIPS)}

Because of the wide range of momenta investigated, numerical calculations are a challenge. Cancellations occur 
in four-vector dot products due to the minus sign, just as they can occur in the calculation of $\Delta t$. With considerable effort, many expressions can be rewritten so large cancellations do not occur. For example, when $\Delta t \geqslant 0$ and $\mathbf{d} \cdot \mathbf{v} \leqslant 0$,

$$
\Delta t=\mathbf{d}^{2} /\left[\sqrt{(\mathbf{d} \cdot \mathbf{v})^{2}+\mathbf{d}^{2} / \gamma^{2}}-\mathbf{d} \cdot \mathbf{v}\right] .
$$

Accurate kinetic energies at any velocity can be obtained from $E-m=\mathbf{p}^{2} /(E+m)$.

Despite careful programming, results obtained with machine long-double precision (64 bits, ca. 20 decimal digits) were suspect for $P>10 \mathrm{~m}$. To untangle real results (e.g., $\eta \neq 1$ ) from numerical accuracy problems, the multiple precision package GMP [3] was adopted. This allows one to choose the number of bits of precision in the calculations dynamically, which can then be repeated and compared at different precisions. Accurate calculation of $\eta-1$, the perpendicular magnetic force, and the net action Eq. (30) require much more than machine precision, We routinely use 128 or 256 bits or occasionally more.

Long after adopting GMP, it was realized that one part of the precision problem had been that the cumulative time coordinate became so large near the turning point (point of closest approach) that $\Delta t$ was very inaccurate when compared to $\Delta \mathbf{r}$. Setting $t_{1}=-|R / V|$ instead of $t_{1}=0$ largely eliminates this problem and restores many orders of magnitude of accuracy to the calculations ( $R$ is the initial distance of the $m=1$ particle from the origin and $V$ is its asymptotic velocity).
How does one parametrize calculational results? The scattering angle relations were inferred from numerical trends and verified computationally.

The determination of this relationship was an iterative process that involved noticing parts of the equations, such as $L \tan (\theta / 2) / \alpha$ being constant as $L$ varied with fixed $P$. This constant product with its proportionality to $\alpha$ might have been surmised from the classical formula Eq. (9). This product was then computed for a wide range of $P$ to guess other factors. For $P \ll m$, where $E \approx m$, the inverse $P$ dependence is apparent. It was easier to guess the remaining factor in the equal mass case.

Graphical presentation of the variability of partial relationships over a large parameter range can be extremely helpful in understanding the remaining variation. When the parametric variation is normalized out, the resulting graph is a straight line over all or part of its range. This approach was used in studying $r(\eta-1)$ for several types of impulses and vertex excitations. It also helped in seeing the angular variation of $\mathbf{r}_{\mathbf{0}} \times \mathbf{F}_{I}$ (after scaling by $\left.r_{o} / u v\right)$.

The conventional Coulomb phase shift formula suggested several parts of my phase shift analysis. When the impulse phase had a logarithmic dependence on $r$, I extracted its coeficient $\Lambda$ and tried standardizing the action to fixed initial and final distances. The quadratic behavior of $\tilde{S}_{0}-\tilde{S}_{L}$ for $L<L_{90}$ and linear behavior for $L>L_{90}$ suggested using $\rho=L / L_{90}$ and ultimately the functional form $\ln \left(1+\rho^{2}\right)$.
[1] J. Huschilt, W. E. Baylis, D. Leiter, and G. Szamosi, Phys. Rev. D 7, 2844 (1973); J. Huschilt and W. E. Baylis, ibid. 13, 3256 (1976).

[2] Roger G. Newton, Scattering Theory of Waves and Particles, 2nd ed. (Dover, Mineola, NY, 2002), pp. 123-132.

[3] Torbjörn Granlund et al., GMP, v. 4.1.4, and later versions, Free Software Foundation, 1994-2011 [http://gmplib.org].

[4] MAXIMA is an open-source alternative to MATHEMATICA [http://maxima.sourceforge.net].

[5] See Supplemental Materal at http://link.aps.org/supplemental/ 10.1103/PhysRevE.84.026611 for a simplified version of the algorithm coded in MAXIMA and for a detailed discussion of cross sections for scattering of a relativistic test particle in a static potential.

[6] Keith R. Symon, Mechanics (Addison-Wesley, Reading, MA, 1953), p. 120.
[7] Claude Itzykson and Jean-Bernard Zuber, Quantum Field Theory (McGraw-Hill, New York, 1980), p. 276ff, and experimental references therein.

[8] L. Landau and E. Lifshitz, The Classical Theory of Fields, translated by Morton Hamermesh (Addison-Wesley, Reading, MA, 1951), pp. 100-102.

[9] Wolfgang Panofsky and Melba Phillips, Classical Electricity and Magnetism (Addison-Wesley, Reading, MA, 1955), p. 289ff.

[10] S. M. Swanson, Phys. Rev. 154, 1601 (1967).

[11] Roger G. Newton, Scattering Theory of Waves and Particles, 2nd ed. (Dover, Mineola, NY, 2002), p. 301, p. 429.

[12] R. P. Feynman, Rev. Mod. Phys. 20, 367 (1948).

[13] M. Sachs and S. L. Schwebel, J. Math. Phys. 3, 843 (1962); Nuovo Cimento 21, Suppl. 2, 197 (1961). Their ideas are in the context of quantum field theory, I have translated them into the classical domain. 